\section{El rol de los gobiernos locales en la conservación de los humedales costeros: el caso del sitio Ramsar Los Pantanos de Villa en Lima, Perú}

Claria Salinas ${ }^{1 *}{ }^{\oplus}$ y Leonor Yarlequé ${ }^{\odot}$
The role of local

governments in the

conservation of coastal

wetlands: the case of

the Los Pantanos de Villa

Ramsar site in Lima, Peru

1 Facultad de Derecho, Pontificia Universidad Católica del Perú. Lima, Perú.

2 Facultad de Derecho de la Universidad Nacional Mayor de San Marcos. Lima, Perú.

\section{SOUTH} SUSTAINABILITY

Citar como: Salinas, C. y Yarlequé, L. (2021). «El rol de los gobiernos locales en la conservación de los humedales costeros: el caso del sitio Ramsar Los Pantanos de Villa en Lima, Perú». South Sustainability, 2(2), e040. doi: https://doi.org/10.21142/SS-02022021-pb002

Artículo recibido: 23/10/2020 Revisado por pares

Artículo aceptado: 25/1/2021

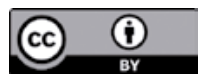

(c) El autor, 2021. Publicado por la Universidad Científica del Sur (Lima, Perú)

${ }^{*} E$-mail de correspondencia: c.salinash@pucp.edu.pe

\section{RESUMEN}

El presente policy brief analiza el rol de los gobiernos locales y municipalidades que tienen jurisdicción en la Zona de Reglamentación Especial de los Pantanos de Villa (ZRE PV) y son miembros del Consejo Directivo de la Autoridad Municipal de los Pantanos de Villa (Prohvilla). Para esto, se llevó a cabo la revisión y el análisis de documentos de gestión como las actas del consejo directivo, los acuerdos de consejo directivo y las memorias anuales, entre otros documentos. De estos, se advierte la poca participación en cuanto a asistencia, votación e iniciativa de algunas municipalidades miembros del consejo directivo, lo cual repercute en la cogestión de la ZRE PV. A partir de ello, se propone la implementación de un reglamento del consejo directivo, mediante el cual se señale las obligaciones, los derechos y demás directrices sobre la participación de cada miembro.

Palabras clave: consejo directivo, conservación, gestión de humedales, gestión municipal de humedales, zona de reglamentación especial

\section{ABSTRACT}

This policy brief analyzes the role of local governments and municipalities with jurisdiction in the Special Regulatory Zone of Pantanos de Villa (ZRE PV) in their participation as members of the Board of Directors of the Municipal Authority of Pantanos de Villa - Prohvilla. In addition, the review and analysis of management documents have been carried out, such as minutes of the Board of Directors, agreements of the Board of Directors, and Annual Reports. The low participation (in terms of attendance, voting, and initiative) of the Board of Directors members has been noticed. Based on what was found, the implementation of a regulation of the Board of Directors is proposed. Through this regulation, the obligations, rights, and other guidelines on the participation of each member are indicated.

Keywords: board of directors, conservation, wetland management, municipal management of wetlands, special regulation area 


\section{Introducción}

El Refugio de Vida Silvestre Los Pantanos de Villa es un área natural protegida (ANP) establecida mediante el Decreto Supremo 055-2006-AG, que cuenta con una superficie de 263,27 hectáreas y se ubica al sur de Lima, Perú. Esta ANP ha sido reconocida como un humedal costero de importancia internacional, sitio Ramsar, debido a su rol como corredor biológico del Pacífico para el recorrido de aves migratorias, la descarga de acuíferos, así como fuente de reserva de biodiversidad y de ostentar un elevado valor social-económico (León, 2019).

Actualmente, existen dos entidades encargadas de la gestión, administración y conservación de este ecosistema. Por un lado, de acuerdo con el Reglamento de la Ley de Áreas Naturales Protegidas, Ley 26834, el Refugio de Vida Silvestre Los Pantanos de Villa pertenece al Sistema Nacional de Áreas Naturales Protegidas y, por lo tanto, su gestión y administración recae en el Sernanp, entidad de competencia nacional. De otro lado, está la Autoridad Municipal de los Pantanos de Villa (Prohvilla), organismo descentralizado de la Municipalidad Metropolitana de Lima (MML), de competencia municipal, que cumple con las funciones de gestión y administración de la Zona de Reglamentación Especial (ZRE) de los Pantanos de Villa, según la Ordenanza 2264-2020-MML (León, 2019).

En tal línea, el presente texto pretende analizar las dinámicas de la gobernanza ambiental, cogestión, que está a cargo de Prohvilla, es decir, de la ZRE de los Pantanos de Villa. En esta ZRE convergen los gobiernos locales: Municipalidad Distrital de Chorrillos, Municipalidad Distrital de Santiago de Surco, Municipalidad Distrital de San Juan de Miraflores y Municipalidad Distrital de Villa El Salvador, ya que cuentan con jurisdicción en la ZRE. A efectos de entender la jurisdicción de cada gobierno local, se presenta la figura 1.

Habiendo señalado que el objeto de estudio recae en la cogestión de la ZRE de los Pantanos de Villa (ZRE PV), se hace necesario precisar en qué consiste esta. Al respecto, de acuerdo con el Reglamento de Acondicionamiento Territorial y Desarrollo Urbano Sostenible de Zonificación del Ministerio de Vivienda, se entiende que son áreas urbanas y de expansión urbana, con o sin construcción, que poseen características de orden físico, ambiental, social o económico, que son desarrolladas urbanísticamente

\section{CHORRILLOS}

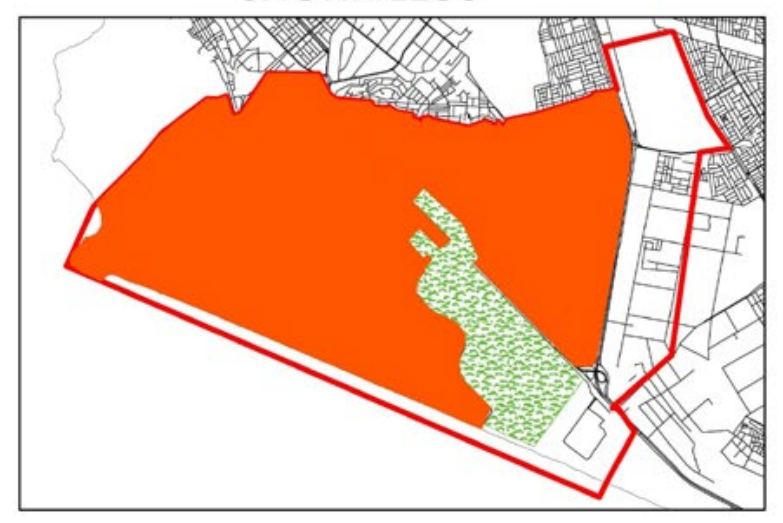

VILLA DE SALVADOR

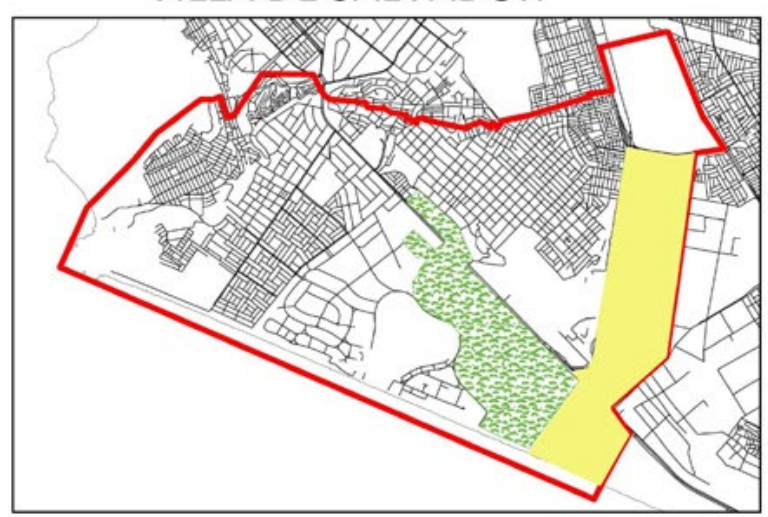

SAN JUAN DE MIRAFLORES

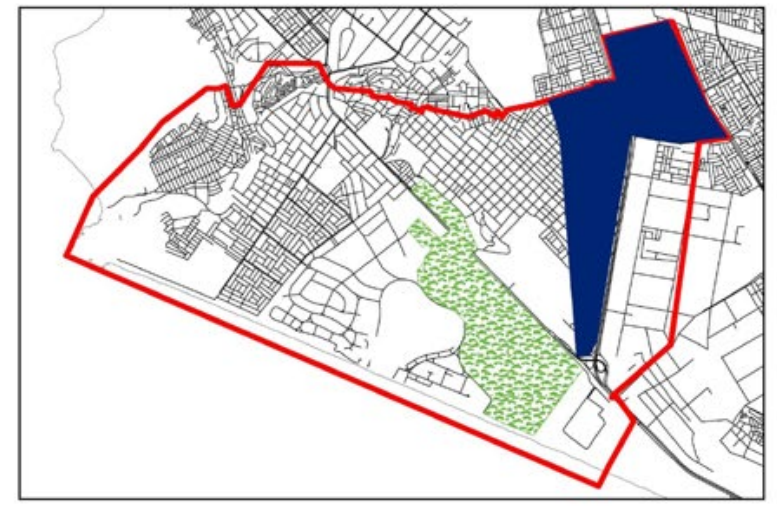

SANTIAGO DE SURCO

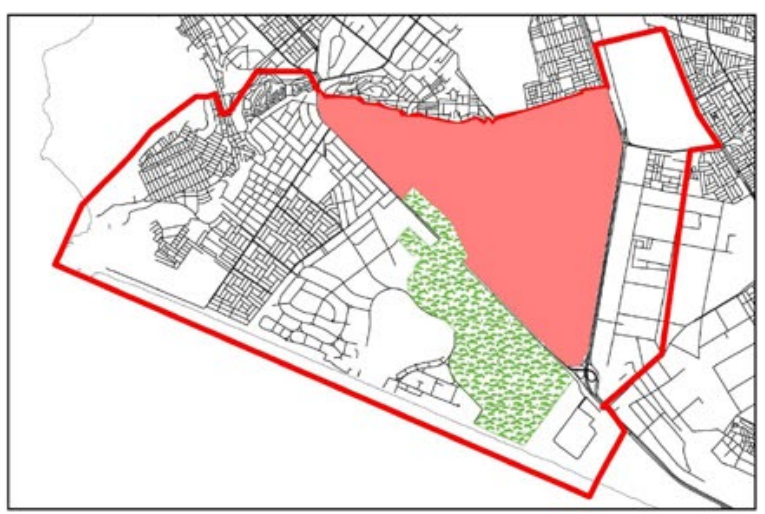

Figura 1. «Gobiernos locales dentro de la Zona de Reglamentación Especial (ZRE) de los Pantanos de Villa». Sombreado en verde el humedal Pantanos de Villa. Delimitado en rojo el perímetro de la ZRE. Demarcada en naranja la jurisdicción de la Municipalidad de Chorrillos, en azul la jurisdicción de la Municipalidad de San Juan de Miraflores, en amarillo la jurisdicción de la Municipalidad de Villa El Salvador y en rosado la jurisdicción de la municipalidad de Santiago de Surco.

Fuente: Oficina General de Asesoría Jurídica (OGAJ) de Prohvilla. 
mediante planes específicos para mantener o mejorar su proceso de desarrollo urbano-ambiental (Decreto Supremo 022-2026-VIVIENDA, 2016).

Para el caso de la ZRE PV, los diferentes documentos normativos y técnicos, a lo largo de los años, la han equiparado con la zona de amortiguamiento, contemplada en la normativa de áreas naturales protegidas. Tal es el caso de la derogada Ordenanza 1845-2014-MML, que indicaba en su artículo IV que «la Zona de Reglamentación Especial Pantanos de Villa (ZRE) es la señalada como Zona de Amortiguamiento (ZA) del Refugio de Vida Silvestre Los Pantanos de Villa». La vigente Ordenanza 2264-2020-MML refiere en su artículo 3 que «la Zona de Reglamentación Especial de los Pantanos de Villa comprende el Humedal Pantanos de Villa y el entorno territorial que ejerce influencia sobre sus procesos ecológicos inherentes. Esta Zona de Reglamentación Especial (ZRE) de los Pantanos de Villa incluye a la Zona de Amortiguamiento (ZA) del Área Natural Protegida que es definida por el Sernanp».

Como se puede apreciar, en ambas ordenanzas asocian la ZRE de los Pantanos de Villa con la ZA, aunque, si bien en la Ordenanza de 2014 se entendía como la misma figura entre ambas, de la vigente Ordenanza 2264-2020-MML se deduce que la ZRE abarca la ZA; por tanto, la ZRE es mayor que la ZA.

Si bien puede resultar confusa la definición tanto de la ZRE como de la ZA, cabe precisar que esta ambigüedad se debe a que el proceso histórico normativo de protección a los Pantanos de Villa no ha sido uniforme; sino que han concurrido distintas normas que regulaban las mismas áreas, zonas de uso de suelo, etc., sin articularse entre ellas (Ojeda, 2016).

Asimismo, si se entiende a la ZRE como la ZA, es necesario indicar la definición de la ZA que, de acuerdo con la Ley de Áreas Naturales Protegidas, Ley 26834, se trata de las «zonas adyacentes al área natural protegida que, por su naturaleza y ubicación, requieren un tratamiento especial para garantizar la conservación del área natural protegida».

Entonces, de todo lo expuesto hasta el momento, destacamos que los Pantanos de Villa se encuentra en cercanía a zonas urbanas altamente concurridas, lo cual dificulta su efectiva conservación. En esa línea, los Pantanos de Villa, al ser reconocidos como sitio Ramsar, cuentan con un estándar internacional de protección, que, como hemos venido desarrollando, se enmarca en la normativa interna en la Ley de Áreas Naturales Protegidas, así como en ordenanzas que competen al gobierno local. También suman al marco normativo la Política Nacional del Ambiente, la Ley General del Ambiente y específicamente la Estrategia Nacional de Humedales, elaboradas por el Ministerio del Ambiente. No obstante, cabe advertir que, en ninguno de los documentos señalados, se hace explícita la tipificación ni la definición de «humedal urbano», que es característico de los Pantanos de Villa.
Esta situación refleja que existe un vacío en la legislación peruana que, si bien puede resolverse con una interpretación sistemática de los diferentes documentos normativos y técnicos, impacta en que los problemas socioambientales que afrontan este tipo de ecosistemas no puedan ser atendidos de forma adecuada y, por tanto, no puedan ser protegidos íntegramente. La inserción de «humedal urbano» como figura de protección conlleva necesariamente a un cambio de paradigma de lo que, hasta el momento, se ha venido entendiendo en las normas por ciudad, planificación territorial, etc., hacia una mirada de urbanismo sostenible.

Retomando la situación de los Pantanos de Villa, en estos persisten problemas socioambientales en sus zonas adyacentes, como contaminación de los canales de agua, invasión de terrenos del área natural protegida, contaminación acústica propias de actividades urbanas que afecta la permanencia de las aves, entre otros (Serrano, 2020). Por ello, es imperativa una adecuada articulación de gestión entre autoridades gubernamentales, cogestión, con el fin de controlar contingencias ambientales no solo del Humedal Los Pantanos de Villa per se, sino también del entorno territorial que ejercen influencia en los procesos ecológicos inherentes al humedal; es decir, de la ZRE.

En tal sentido, para abordar en el estudio de la gestión de la ZRE PV, rectoría a cargo de Prohvilla, se analizó el consejo directivo, que es el órgano máximo de la institución y que tiene la misión de gestionar, concertar, formular, actualizar y evaluar los planes de gestión y manejo ambiental de la ZRE (artículo 7 ROF de Prohvilla). Este consejo directivo está conformado por nueve miembros: la $\mathrm{MML}$, en la presidencia; Prohvilla, en la Secretaría Técnica; el Servicio de Parques de Lima (Serpar), el Sernanp, el Instituto Metropolitano de Planificación (IMP), y las cuatro municipalidades anteriormente mencionadas, que cuentan con jurisdicción en la ZRE (Municipalidad de Chorrillos, Municipalidad de Santiago de Surco, Municipalidad de San Juan de Miraflores y Municipalidad de Villa El Salvador).

\section{Enfoques y discusión}

En el presente manuscrito se ha tomado un enfoque de gestión pública desde la mirada del derecho administrativo y enmarcado en la gobernanza ambiental. Respecto a gobernanza, es un modelo de organización social descentralizado y participativo para la toma de decisiones que, en términos ambientales, busca un equilibrio entre la conservación de la naturaleza y el desarrollo de los territorios. Este concepto promueve la adopción de decisiones de abajo hacia arriba (bottomup), para que los gobiernos locales puedan desarrollar la capacidad de diseñar y gestionar iniciativas de acuerdo con sus necesidades locales. En este sentido, se busca que las instituciones locales sean capaces de gobernarse a sí mismas y de gestionar sus propios ecosistemas de humedales, realzando el valor del conocimiento local 
como mecanismo de protección (Muñoz-Lobos et al., 2020).

Ahora bien, la cogestión es un tipo de gobernanza, entendido como un proceso de toma de decisiones compartido entre los actores que hacen uso de los recursos y los administradores formales para establecer las políticas de gestión de uso del área protegida; es decir, se trata de una gobernanza conjunta (León, 2019).

En tal línea, Prohvilla, a través de su consejo directivo, cumple la función de articular los mejores intereses y decisiones para la protección de la ZRE PV; la pluralidad de miembros que conforman el consejo directivo representa distintos niveles de gobierno (nacional, local y organismos descentralizados).

Asimismo, este consejo directivo es un órgano colegiado de mayor nivel jerárquico. Al respecto, la Ley de Procedimiento Administrativo General, Ley 27444, en sus artículos del 106 al 113, señala que los órganos colegiados forman parte de la estructura organizativa de la administración pública en los que la toma de decisiones responde a un acuerdo de naturaleza colectiva. Son ejemplos de órganos colegiados aquellos de nivel directivo como los consejos directivos (Guzmán, 2004).

De igual forma, la doctrina administrativa resalta como naturaleza jurídica de los órganos colegiados la horizontalidad entre sus miembros. No hay subordinación

a) 12

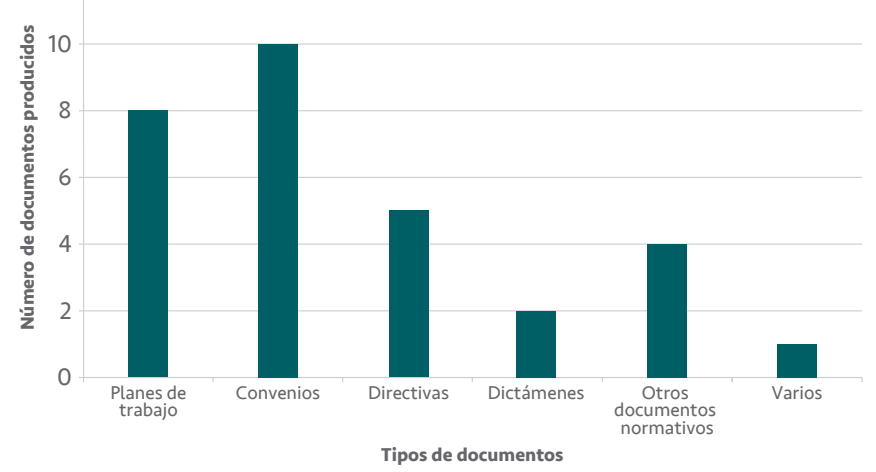

c)

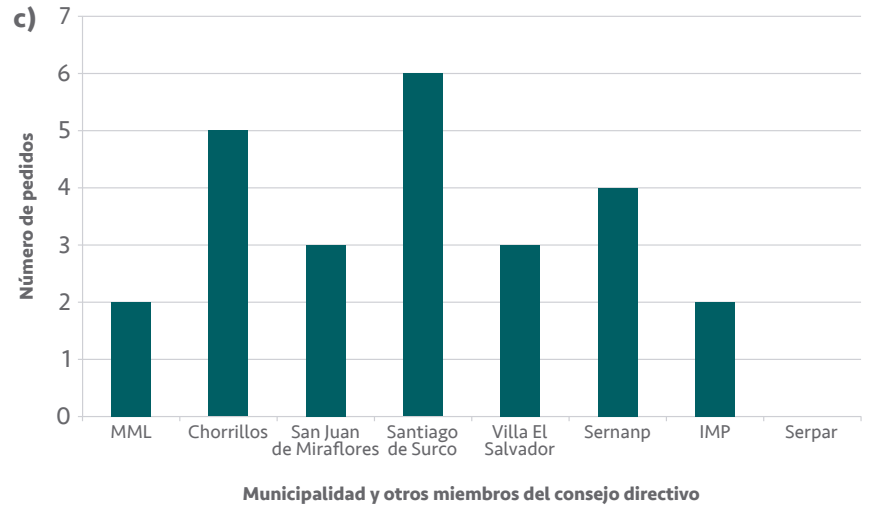

jerárquica, pues estos concurren en una situación de igualdad en la formación de la voluntad del órgano colegiado, a excepción de empates en la votación de decisiones, en los que el miembro que se desempeñe como presidente decidirá con su voto el desenlace del empate. Asimismo, el proceso de gestación y culminación del acto administrativo que emite el órgano colegiado consta de cinco etapas: (i) nombramiento, (ii) convocatoria, (iii) deliberación, (iv) quórum y (v) votación (Méndez y Sandoval, 2018). De lo señalado, se puede concluir que un consejo directivo es un órgano colegiado en el que se determinan las decisiones de acuerdo con la mayoría; es decir, es una voluntad colectiva de la mayoría (quórum).

Debido a la naturaleza jurídica del consejo directivo de Prohvilla, todos los miembros que lo conforman participan bajo un enfoque de horizontalidad e igualdad, a pesar de que entre sus miembros confluyan competencias nacional y local, y materializan su participación a través del voto que realizan en cada asamblea ordinaria o extraordinaria. No obstante, el miembro que cumple el rol de la presidencia, como en el caso de la MML, tiene una participación diferente en situaciones de empate, pues su voto se constituye como el voto decisivo. Por tanto, debe entenderse que los miembros del consejo directivo cumplen con su participación en la cogestión mediante la asistencia, votación y participación en sí con opiniones, propuestas, identificación de prioridades, concertación de soluciones, etc., en las asambleas.
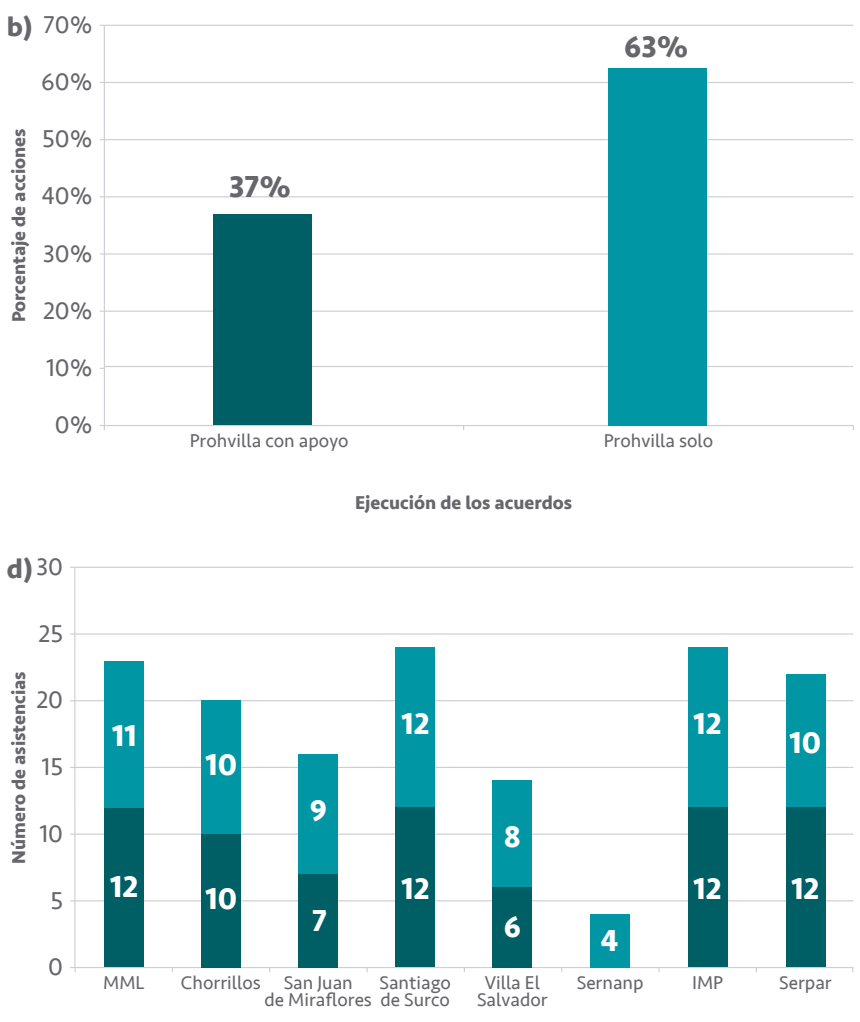

Municipalidad y otros miembros del consejo directivo

Figura 2. Resultados del análisis de las actas de consejo directivo 2019-2020: a) tipos de documentos aprobados por el consejo directivo 2020; b) colaboración de las municipalidades miembros del consejo directivo a Prohvilla en la ejecución de acuerdos de 2019; c) solicitudes y pedidos de los miembros del consejo directivo de 2019; y d) asistencias del consejo directivo 2019-2020. 
En sus asambleas, el consejo directivo aprueba documentos de gestión interna, como la firma de convenios, la iniciativa de los órganos de línea de Prohvilla, el POA, el calendario anual e iniciativas acordes con la situación laboral, entre otros. Pero también aprueba decisiones o dictámenes que competen actividades conjuntas con los municipios locales y con los demás miembros; por ejemplo, las actividades que incentiven la conservación del ANP y los dictámenes sobre el cambio de zonificación a pedido de algún administrado, entre otros. En tal línea, para el presente trabajo se elaboró una matriz de consistencia sobre los acuerdos, evidencias y decisiones del consejo directivo de Prohvilla, al ser este el órgano mediante el cual las municipalidades tienen participación directa para la gestión de la ZRE PV. Esta información se presenta en la figura 2.

Una búsqueda sistemática en la información y documentos revisados, principalmente, acuerdos del consejo directivo de 2020, revelaron que la mayoría de los acuerdos son convenios de colaboración interinstitucional, seguidos por planes de trabajo (figura 2a).

Tanto los convenios como los planes de trabajo son documentos que coadyuvan en la realización de diferentes actividades a nivel interno de la institución, lo cual refleja que es mínima la existencia de planes de trabajo o actividades a un nivel de articulación con los demás miembros. No obstante, el hecho de que estos instrumentos de gestión interna de Prohvilla sean discutidos para su posterior aprobación por el pleno del consejo directivo indica también transparencia para la toma de decisiones de estos procesos, así como el involucramiento de los demás miembros del consejo.

Asimismo, en 2019 se realizaron 12 asambleas y de cada una se tiene un acta de consejo directivo en la que se plasman los acuerdos. Sobre ello, se observa que, de todos los acuerdos en ese año, el $63 \%$ no requirió de la colaboración de las municipalidades o demás miembros del consejo directivo para su ejecución y el $37 \%$ sí requirió la colaboración de las municipalidades y demás miembros (figura 2b).

El hecho de que en un mayor porcentaje no se haya requerido la colaboración de las municipalidades evidencia también la falta de presupuesto asignado específicamente para la articulación en la gestión de la ZRE PV, por parte de las municipalidades. Ello se puede notar debido a que en ninguna de las municipalidades existe una oficina o unidad específicamente enfocada en la articulación para la gestión de la ZRE.

Respecto a la participación de los miembros del consejo directivo, en 2019 , en relación con las solicitudes y pedidos que se realizaron en las asambleas, se advierte que el número máximo de solicitudes fue hecho por la Municipalidad de Santiago de Surco, seguida por la Municipalidad de Chorrillos, Sernanp, y, después, la participación de las Municipalidades de San Juan de
Miraflores y Villa El Salvador; en penúltimo lugar, la MML y el IMP; por último, con ninguna participación, Serpar (figura 2c).

Las solicitudes y pedidos del Consejo Directivo son relevantes en la cogestión de la ZRE PV; en el sentido de que representan la participación de cada miembro en la dirección, organización y administración de las áreas que tienen en su jurisdicción. Además de ello, el consejo directivo, al ser un órgano colegiado, responde a una lógica de la mayoría. Por lo tanto, es importante la participación para que se puedan tomar en cuenta las opiniones e información que cada miembro presente, con el fin de ejecutar las mejores decisiones.

Por último, en relación con las asistencias a las asambleas del consejo directivo, en 2019 y 2020, las cuales representan al voto de cada miembro sobre las solicitudes y pedidos hechos en cada sesión, se encontró la siguiente información (figura 2d):

- En 2019 la Municipalidad de Santiago de Surco y el IMP fueron los miembros con mayor asistencia a las asambleas; seguidas por la MML; luego está la Municipalidad de Chorrillos y Serpar; después la Municipalidad de San Juan de Miraflores; y, en penúltimo y último lugar, la Municipalidad de Villa El Salvador y Serpar, respectivamente.

- En 2020 los miembros con mayor asistencia fueron el IMP, Serpar, la MML y la Municipalidad de Santiago de Surco; seguidos por la Municipalidad de Chorrillos; luego, la Municipalidad de San Juan de Miraflores; después, la Municipalidad de Villa El Salvador y, por último, Sernanp.

De la sistematización de esta información, se evidencia en que no todos los miembros asisten a las asambleas ordinarias de consejo directivo, que se realizan una vez al mes. Sobre ello, cabe precisar que, si bien los años de estudios abordados fueron 2019 y 2020, en este último apareció la emergencia sanitaria por la covid-19, por lo que no es causal a que no se hayan reunido mensualmente. Las asambleas del consejo directivo en el contexto de la pandemia se hicieron por medios virtuales; a pesar de ello, se nota la falta de asistencia de algunos miembros. En tal sentido, se debe tener presente que, en los órganos colegiados, como un consejo directivo, parte del proceso de consolidación del acto administrativo es el quórum y la votación, en los que es indispensable la asistencia y participación de sus miembros. El objetivo de ello es que los acuerdos del consejo directivo plasmen la voluntad colectiva de sus miembros. Sin embargo, ante la poca asistencia y compromiso, esta voluntad colectiva, como la finalidad del órgano colegiado, podría verse tergiversada.

Por otro lado, como experiencia cercana de salvaguarda de humedales en contextos urbanos se conoce el caso de los humedales urbanos de Chile. En enero de 2020 el gobierno chileno promulgó la Ley de Humedales Urbano 21.202, que ha sido caracterizada como pionera en materia ambiental 
en la región. Con esta ley, Chile hace un reconocimiento explícito a la protección de los humedales urbanos y a su definición. Ello ha resultado una modificación en la Ley General de Urbanismo y Construcción de Chile y en sus instrumentos de planificación territorial, que ahora deben tomar en cuenta la protección de los humedales urbanos (Rojas, 2020). Al asociar esto con el caso peruano, se nota que en el Perú, al no contar con una protección explícita sobre «humedal urbano», aún no se ha podido dar este cambio de paradigma hacia el urbanismo sostenible que se desarrolló en la primera parte de este texto.

Asimismo, de la experiencia chilena se advierte que la protección a los humedales urbanos son en general a todos los humedales en esa situación y no solo a los reconocidos como sitios Ramsar. Para el contexto peruano, tener una ley específica de humedales urbanos fortalecería la protección de los Pantanos de Villa, sitio Ramsar, pero también de otros humedales que no han sido reconocidos Ramsar. Además de ello, se debe tener en cuenta que el reconocimiento explícito en la ley va de la mano con mayor voluntad política, designación presupuestaria, entre otros aspectos.

Respecto al modelo de gestión que señalan la Ley de Humedales Urbanos de Chile y demás normas, se advierte que se otorga a los gobiernos comunales, municipios, la potestad de gestionar la protección, conservación y preservación de los humedales urbanos ubicados en su comuna. De igual forma, promueve que el Ministerio del Medio Ambiente de Chile cree comités a nivel nacional, regional y comunal para promover la adecuada gestión y gobernanza de los humedales urbanos, que permita la participación efectiva de los actores involucrados (Mallega, 2020).

\section{Conclusiones y recomendaciones}

La participación de los gobiernos locales que tienen jurisdicción en la ZRE PV se plasma en su participación como miembros del consejo directivo de Prohvilla. No obstante, se ha evidenciado que no todas las municipalidades tienen una participación activa en las asambleas del consejo directivo. Esto repercute en la toma de decisiones del consejo directivo, que refiere en la práctica a la cogestión de la ZRE.
Esta situación se debe, en parte, a la falta de directrices, específicamente, de un reglamento que detalle la designación de los miembros del consejo directivo, sus obligaciones, derechos, tipos de documentos que se aprueban, la convocatoria de las asambleas, el quórum y las sanciones, entre otros asuntos. Conforme a la investigación realizada, el consejo directivo de Prohvilla, hasta el momento, no ha tenido un reglamento en el que se estipulen todos los temas mencionados.

Por ello, consideramos la necesidad e importancia del reglamento del consejo directivo de Prohvilla para garantizar una eficiente cogestión de la ZRE. Asimismo, consideramos necesario incentivar la promulgación de una Ley de Humedales Urbanos o, en todo caso, un reconocimiento explícito en los documentos técnicos y normativos al «humedal urbano» por presentar este un contexto distinto que para su protección efectiva debe ser abordado también desde otros sectores, como los de planificación territorial y urbanismo. De igual forma, debe incentivarse en cada municipalidad que tiene jurisdicción en la ZRE PV la participación en la conservación de la ZRE, ya sea mediante el reconocimiento de buenas prácticas, el fortalecimiento en la gestión ambiental a sus recursos humanos, entre otros.

\section{Agradecimientos}

A Prohvilla, por la oportunidad de participar del programa de pasantía «Gestión pública y conservación de humedales 2021-|». Asimismo, a la Oficina General de Asesoría Jurídica de Prohvilla, por su apoyo y disposición para la elaboración del presente escrito.

\section{Contribución de autoría}

Ambas autoras contribuyeron de igual manera en la recopilación y el análisis de la información, y en la elaboración y la redacción del manuscrito.

\section{Fuente de financiamiento}

Autofinanciado y en colaboración con Prohvilla.

\section{Potenciales conflictos de interés}

Las autoras declaran no tener conflicto de intereses. 


\section{Referencias bibliográficas}

Guzmán, C. (2004). La administración pública y el procedimiento administrativo general. Lima: Editorial ARA.

lannacone, J. y Alvariño, L. (2007). «Diversidad y abundancia de comunidades zooplanctonicas litorales del humedal Pantanos de Villa». Gayana, 71(1), pp. 49-65. Disponible en: https://scielo.conicyt. $\mathrm{cl} / \mathrm{pdf} /$ gayana/v71n1/art06.pdf

León, G. (2019). Gobernanza ambiental y conservación: las gestiones del SERNANP y PROHVILLA en el Refugio de Vida Silvestre los Pantanos de Villa, y las acciones para su conservación. Tesis de maestría en Desarrollo Ambiental. Pontificia Universidad Católica del Perú. Disponible en: https://tesis.pucp.edu.pe/repositorio/ handle/20.500.12404/14122

Mallega, M. (2020). Humedales urbanos: historia de una ley pionera y ciudadana de protección ambiental. Disponible en: http:// bibliotecadigital.ciren.cl/bitstream/handle/123456789/29473/ obtienearchivo.pdf? sequence=1\&isAllowed $=y$

Méndez, L. y Sandoval, C. (2018). Los órganos colegiados: impacto en la gestión de la administración pública local. Tesis de grado en Derecho. Universidad Nacional de Trujillo. Disponible en: https://dspace. unitru.edu.pe/handle/UNITRU/11001\#: :text=Se\%20precisa\%20 por $\% 20 \%$ C 3\%BAltimo \% 2C\%20que,colegiados $\% 2$ C 20 protegiendo\%20la\%20legalidad\%2C\%20una

Ministerio del Ambiente. (2015). Decreto Supremo 004-2015-MINAM. Estrategia Nacional de Humedales (ENH). Disponible en: https:// www.minam.gob.pe/wp-content/uploads/2015/01/AnexoDecreto-Supremo-N\%C2\%B0-004-2015-MINAM2.pdf

Muñoz-Lobos, C., Vásquez, A. y Cortés-Donoso, E. (2020). «El rol de los gobiernos locales en la gobernanza de protección de humedales. El caso del humedal de Pichicuy (Chile)». Urbano (Concepción), 23(42), pp. 98-111. Disponible en: https://scielo.conicyt.cl/scielo. php?pid=S0718-36072020000200098\&script=sci_arttext\&tlng=e
Ojeda, R. (2016). Evaluación de la contaminación acústica ambiental en el área natural protegida «Pantanos de Villa». Tesis de grado en Ingeniería Ambiental. Universidad Nacional Federico Villarreal. Disponible en: http://repositorio.unfv.edu.pe/handle/UNFV/1490

Prohvilla, Autoridad Municipal de los Pantanos de Villa. (2019) Plan Anual 2019. Disponible en: http://prohvilla.munlima.gob.pe/ transparencia/plan-de-trabajo-2019.pdf

Prohvilla, Autoridad Municipal de los Pantanos de Villa. (S. f.) Zona de Reglamentación Especial. Disponible en: http://prohvilla. munlima.gob.pe/zonificacion.html

Rojas, C., Sepúlveda-Zúñiga, E., Barbosa, O., Rojas, O. y Martínez, C. (2015). «Patrones de urbanización en la biodiversidad de humedales urbanos en Concepción metropolitano». Revista de Geografía Norte Grande, (61), pp. 181-204. Disponible en: https://www.redalyc.org/ pdf/300/30041119010.pdf

Rojas, C. (2020). «Humedales urbanos en Chile: Impacto en políticas públicas y el Desarrollo Sostenible». En M. Mallega (Ed.), Humedales urbanos. Historia de una ley pionera y ciudadana de protección ambiental (pp. 42-50). Disponible en: http://bibliotecadigital. ciren.cl/bitstream/handle/123456789/29473/obtienearchivo. pdf? sequence $=1 \&$ is Allowed $=y$

Serrano, D. (2020). Recuperación y habilitación de la zona de amortiguamiento de los Pantanos de Villa y diseño de un centro de educación ambiental. Trabajo de investigación de bachiller en Arquitectura, Urbanismo y Territorio. Universidad San Ignacio de Loyola. Disponible en: http://repositorio.usil.edu.pe/bitstream/ USIL/9925/3/2020_Serrano\%20Pazos.pdf

Solano, P. (2013). «Legislación y conceptos aplicables a las áreas naturales protegidas en el Perú». Derecho PUCP, 70, pp. 143-164. Disponible en: https://www.redalyc.org/pdf/5336/533656139009. pdf 